\title{
Smart Financial Management System Based on Data Ming and Man-Machine Management
}

\author{
Maotao Lai \\ Finance Department, Minxi Vocational \& Technical College, Longyan 364000, China \\ Correspondence should be addressed to Maotao Lai; 82003001@lyun.edu.cn
}

Received 28 October 2021; Revised 27 November 2021; Accepted 29 November 2021; Published 5 January 2022

Academic Editor: Xin Ning

Copyright ( 2022 Maotao Lai. This is an open access article distributed under the Creative Commons Attribution License, which permits unrestricted use, distribution, and reproduction in any medium, provided the original work is properly cited.

\begin{abstract}
To begin, the architecture of an intelligent financial management system is thoroughly investigated, and a new architecture of an intelligent financial management support system based on data mining is developed. Second, it goes over the definition and structure of a data warehouse and data mining, as well as how to use data mining strategy and technology in financial management. Data mining in relation to technology is being investigated, as is the development of an intelligent data mining algorithm. The flaws of the intelligent data mining algorithm are discovered through an analysis and summary of the algorithm, and an improved algorithm is proposed to address the flaws. Related mining experiments are carried out on the improved algorithm, and the experiment shows that it has certain advantages. Then, using an intelligent forecasting financial management decision as an example, the intelligent financial management based on data mining is thoroughly investigated, the basic design framework for intelligent financial management is established, and the application of a data mining model in decision support system is introduced.
\end{abstract}

\section{Introduction}

The continuous development of artificial intelligence technology, computer technology, and financial knowledge model makes the realization of many complicated financial models or analysis and prediction methods become simple and feasible. These are for enterprise financial management innovation provides a powerful technical support, to make the function of accounting information system from accounting development into management decision-making model, even using modern computer technology to establish effective smart financial management support system is an effective way, is also an inevitable trend of the development of financial informatization [1].

Scientific analysis and management of the business mode, cost control, financial status, and other aspects of the enterprise, based on the scientific analysis of all kinds of data, targeted formulation of corresponding decisions and measures, to promote the enterprise can become bigger and stronger. But with so much business data, and especially financial data, how do we discover the information hidden in that data? Are there necessary relationships between various types of financial data? For example, is the expense of the enterprise reasonable? Does the enterprise face financial risk? Due to the continuous development of information processing technology, complex financial problems in the past have become easy to handle by establishing accounting models and methods [2]. Data mining is to find hidden in a large amount of data and valuable knowledge and information, artificial intelligence technology is used by simulating specialist knowledge and logic for complex problem solving and reasoning ability, and these new techniques for financial analysis, accounting information system, and financial intelligence provide powerful technical support. Using these technologies, an intelligent system of operation and financial management analysis based on knowledge data can be established, which can transform the accounting information 
system from accounting type to operation decision-making type, and provide intelligent human-computer interaction information system helpful for management and decision makers [3].

Through the study of data warehouse, data mining theory, and technology, combined with their own practical work on the basic framework of intelligent financial management support system, the realization of the function and the adoption of key technologies are studied and discussed. The study of three key technologies in the intelligent financial management support system is clustering method, decision tree method, and association rule mining technology. After analyzing the characteristics and advantages of these methods, the application of these three key technologies in specific modules is studied. Moreover, the association rule mining algorithm is improved to improve its efficiency. The data mining technology applied in financial management and the intelligent data mining algorithm are analyzed and studied, and its improved algorithm is proposed.

\section{Related Work}

In foreign countries, data mining technology because of its research time and field is relatively early, and researchers have made a great breakthrough after a period of accumulation, developed a more mature algorithm, formed a more commonly used data mining tools, and set up some wellknown research institutions [4]. At present, relatively mature data mining tools can be roughly divided into two types, one is similar to SPSS, SAS, LBS Capital Management, and other Management software, and the other is the use of relatively popular new technologies, such as decision tree algorithm, genetic algorithm, artificial neural network, expert system technology, and other tools, such as Neural network Browser, CBR, Fidelity Stock Selector, SPSS Clementine, and other tools [5, 6]. Some researchers who have studied data mining for a long time have put forward influential mining algorithms, such as the concept tree promotion algorithm [7], classification algorithm [8], and association algorithm, attracted extensive attention of many researchers, and have been improved and derived on the basis of this algorithm $[9,10]$. In addition, the data mining technology has also caused the extensive concern of the authority of some well-known research institutions, the institutions involved in institutions of higher learning and independent research institutes. Using modern high technology of huge amounts of information automatic analysis and processing method of research, in order to improve smart financial management analysis methods of intelligence, such as [10] based on rough set theory, an intelligent decision analysis algorithm for financial management is designed, which can carry out information association. The algorithm can satisfy the basic theorem of probability theory and Bayes' theorem and can automatically draw analysis conclusions from a large number of unstructured data. In view of the situation that $80 \%$ of the information in an organization is hidden in files [11], the concept of document warehouse based on business intelligence is proposed, and the corresponding algorithm is designed. At the same time, the combination of document warehouse and data warehouse can realize the intelligent analysis of organizational business data by using OLPA technology. In view of the large amount of CRM(customer relationship management) and CDR(customer distribution map) data in telecom operators [12], a service-oriented business intelligence architecture based on decision tree algorithm is developed to facilitate the intelligent analysis of a large amount of data by telecom operators.

The accounting information provided by traditional accounting treatment and financial analysis methods could not meet the needs of people to analyze and make decisions on complex problems in form, timeliness, and effectiveness. With the rise of artificial intelligence and expert system, effective methods are provided for solving such problems. In addition, it promotes the development of modern accounting system from information and network to intelligence [13, 14]. Also known as the three-part theory, it became the basic theory of DSS and played a very important role in the later development of DSS. The three-system structure of language system, problem processing system, and knowledge system has certain characteristics in problem processing, which makes it have a good influence and lays the foundation for the development of intelligent financial management system [15]. After a long time of theoretical and applied research, great progress has been made in application. For example, IFPS "conversational financial planning system" [16] is a conversational system that can support managers' planning and decisionmaking, mainly for middle and senior decision-making. In AAIMS system, this system is used for planning, finance, and various plan optimizations, benefit analysis, plan forecasting, and so on. In recent years, after the emergence of data warehouse technology, FDSS has more powerful data analysis and decision support functions by combining it with online analytical processing functions [17]. Data mining is derived from the field of artificial intelligence and is the product of machine learning and database technology. In its research and practice progress, gradually integrate artificial intelligence, intelligent information processing, semantic Web, and other technologies and constantly improve the level of simulating human intelligence. With the indepth development of data mining research and application, intelligent information processing technology is introduced, which promotes the further intelligent development of data mining technology. Intelligent information processing is the product of the combination of artificial intelligence technology and information technology, which extensively simulates human intelligence to deal with various complex information, including unstructured information, massive information, incomplete information, uncertain information, fuzzy information, multimedia information, time and space information, cognitive information, and knowledge information $[18,19]$. Intelligent information processing technologies and methods mainly include mathematical statistics, fuzzy information processing, rough set information processing, neural network information processing, evolutionary computing, and large-scale intelligent information processing technologies based on information and knowledge management, such as information analysis, classification, intelligent retrieval, and intelligent search. 
The ideas and algorithms of these technologies provide new ideas for the development of data mining and effectively deal with the problems that are difficult or impossible to be dealt with by traditional statistical analysis and machine learning techniques, especially the analysis and prediction of high-dimensional nonlinear random, dynamic, or chaotic system behavior [20, 21]. At present, the mining method based on ontology is mainly includes three aspects: one is based on the concept of text mining, such as constructed to describe the semantic ontology model grid data [22], on the basis of the text mining process is discussed to construct methods: application of ontology and semantic Web technology to build a Web text classifier [23] and automatically realize automatic classification based on semantic Web text information. The second is based on the mining of relationships between concepts. For example, aiming at the problem of lexical disambiguation and structural disambiguation in phrase analysis of natural language processing system [24], the method of obtaining Chinese semantic collocation rules based on association analysis mining is studied. The third is mining based on conceptual semantic analysis. For example, semantic analysis technology is used to discover semantic architecture from Web log data [25], and experiments prove that semantic architecture automatically extracted based on semantic analysis technology that has significantly improved effect after it is used in log data mining. At present, researches on dynamic data mining mainly focus on data flow mining, fractal data mining, online analysis mining, empirical mode decomposition, and connection discovery technology [26]. Although the application of intelligent financial system has achieved good development and results, there are still many defects in the current application, limited to the diagonal description of the current situation of the enterprise, and can only be regarded as the application stage of financial analysis. The research on integrating expert knowledge into software system to solve the prevalent problems of enterprise management and decision-making is only at the research stage.

\section{Data Mining Intelligent Financial Management System Architecture}

3.1. Architecture of Intelligent Financial Management System. Intelligent Decision Support System (IDSS) is formed by the integration of Decision Support System (DSS) and expert system (ES). Decision support system is mainly composed of model base, human-computer interaction system, and database system. Expert system is mainly composed of inference machine, dynamic database, and knowledge base. The structure of the intelligent financial management support system integrated by the two is generally shown in Figure 1.

The common foundation of the new architecture is the database. The new structure includes two topics, one is knowledge base system and model base system, which provides auxiliary decision information combining qualitative analysis (knowledge reasoning) and quantitative analysis (model calculation) for decision problems. The other subject is data warehouse and data mining; it extracts useful infor- mation and knowledge from database and data warehouse, and these information and knowledge reflect the inherent law of a large number of data.

3.2. Data Mining Technology and Its Application in Intelligent Financial Management. The intelligent financial management support system developed by the use of data warehouse and data mining technology, using relational data storage format to store massive business subject data, enhance the ability of large data volume system management expansion. It uses parallel processing technology to deal with complex query request service, realizes decision support query optimization, collects information from a large amount of enterprise data, and provides statistical trend analysis and forecast report flexibly and interactively by using mathematical model and data processing technology. The system also establishes a variety of data mining models, which can automatically analyze the data, perform inductive reasoning and association on the data in the data warehouse, find some internal associations between the data, and discover potential patterns that play a very important role in information prediction and decision-making behavior, so as to establish a new business model. In order to help decision-makers to develop market strategy, make the right decision. It is divided into three layers, data acquisition layer, data storage organization layer, and data analysis and display layer, as shown in Figure 2.

3.2.1. Define the Problem. Define the management problem to be solved and determine the target of data mining.

3.2.2. Data Preparation. Data mining is built on the basis of data warehouse; so, only good data basis can get the ideal mining results. Therefore, it is very important to conduct necessary data processing before starting data mining. Data preparation generally includes data selection-find the data set needed for data mining in the data warehouse and data preprocessing-in order to ensure the integrity and consistency of data, and data should be processed twice, such as adding lost items, removing noise, and deleting invalid data.

3.2.3. Build a Model. This is the most critical step, according to the characteristics of the data or data type to choose the appropriate algorithm, after purification and transformation of the data set, select the appropriate parameters and data mining and establish the model.

3.2.4. Evaluate Interpretation Models. Interpret and evaluate the established model and translate it into a language that can be understood by users. If the model is reasonable, it can be used experimentally. If problems are considered, the process needs to be repeated until satisfactory results are obtained.

3.2.5. Use and Consolidate the Model. Put the final model into practice and monitor its performance, making adjustments as appropriate to adapt to actual changes. 


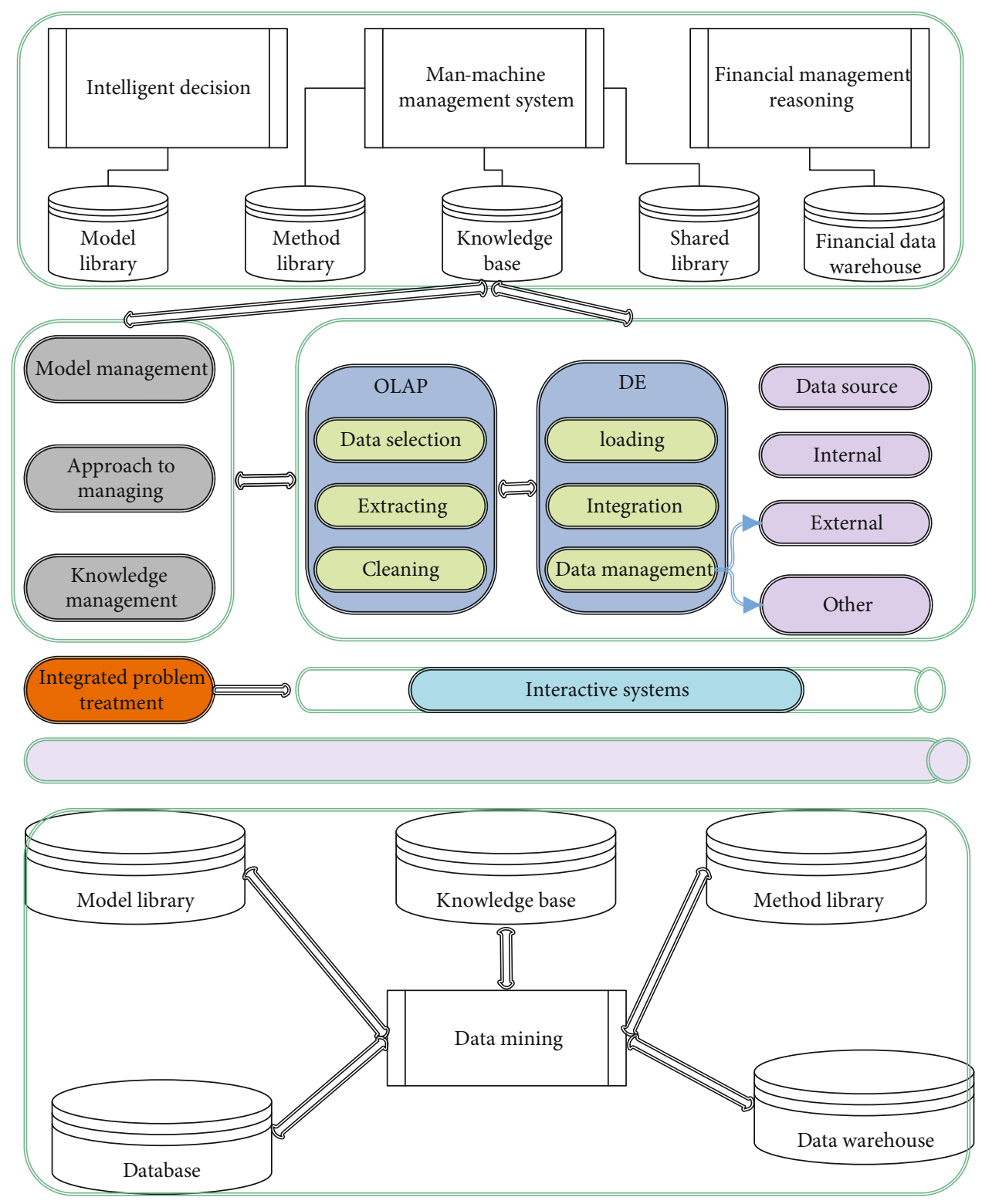

FIGURE 1: New architecture diagram of intelligent financial management support system.

\section{Research on Data Mining Algorithm of Intelligent Financial Management System}

How to find the optimal partition method for the sample requires a function to determine which problems achieve the most balanced partition, and this requires that the information gain can be achieved. When the intelligent data mining algorithm selects attributes, the one with the most information gain is first selected as the test attribute of the current node, which can minimize the information of sample classification in the result classification. And try to make sure you find a simple tree that describes the information in the sample set. The derivation of the formula is described below. The core of ID 3(Iterative Dichotomiser 3) algorithm is in the selection of node attributes of the decision tree, and the information gain is taken as the basis for selection. When testing each nonleaf node, the branch of the smallest point of the tested record can be measured until all subsets contain only the same type of data. Finally, a decision tree is obtained, which can realize the classification of new samples.

The method of calculating the information gain of attributes is as follows. By calculating the information gain of each attribute and comparing its size, it is not difficult to obtain the attribute with the maximum information gain. Let us say we have $S$ sample sets. Assuming that the number of different values of the attribute of class label $p$ is $m$, then the definition that $m$ are of the same kind is

$$
T\left(s_{1} \cdots s_{m}\right)=\sum_{i=1}^{m} P_{i} \log _{2} P_{i} .
$$

In the design of the index system, it is necessary to consider all the factors from the above aspects. Through the 


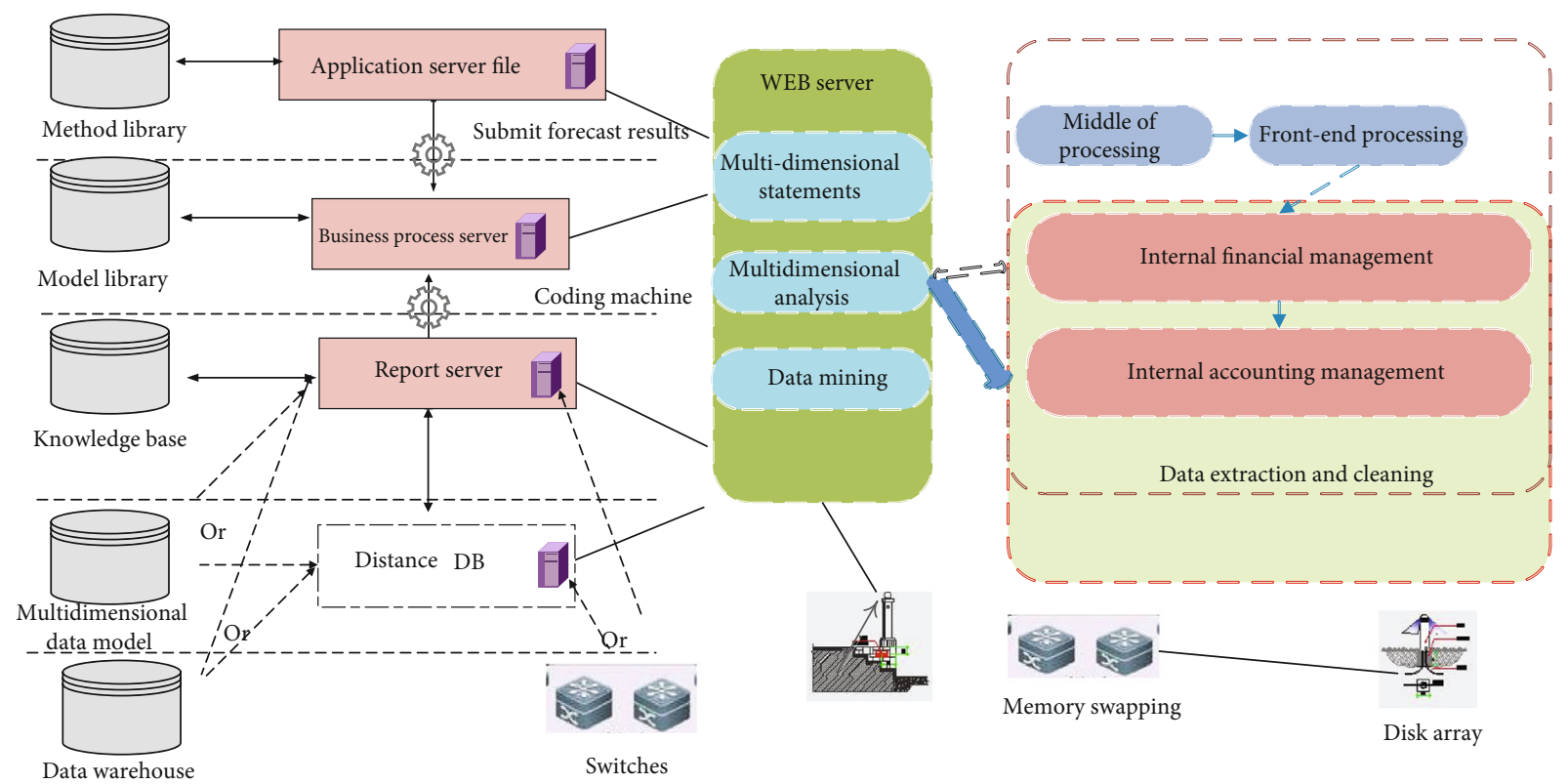

FIGURE 2: Intelligent management research structure of data mining.

TABLe 1: Comprehensive evaluation index system of intelligent financial management scheme.

\begin{tabular}{l}
\hline Indicators category \\
Financial indicators \\
Technological innovation \\
level \\
Development potential level \\
indicators
\end{tabular}
indicators

Business process level metrics

Margin smart financial management, financial net present value, internal rate of return, payback period of static smart financial management, dynamic payback period of smart financial management, revised economic value added, return on intellectual capital, capital preservation rate of increment rate, sales net interest rate, cost reduction, current assets turnover, accounts receivable turnover, assets and interest rate and interest rate, cost, profit margin, net, net assets cash flow, debt yield, cost and expense reduction rate.

Internal customer (employee) indicators: employee satisfaction, employee work efficiency, employee dimission rate, employee suggestion rate, employee training rate, employee working ability, employee suggestion ability; external customer indicators: Market share, customer retention rate, customer profitability, customer acquisition rate, product timely delivery rate, sales increase or decrease rate of existing customers, return rate

Growth rate of sales, growth rate of net equity, growth rate of assets growth rate of contribution value of intellectual assets growth rate of contribution value of knowledge and intellectual assets, growth rate of net profit, renewal rate of fixed assets, intellectual capital ratio, three-year average growth rate of sales, threeyear average growth rate of capital, ratio of technology $r \& d$ (research and development) investment.

Product life cycle, product production turnover efficiency, timely troubleshooting rate, product qualified rate, accident rate, logistics safety rate, equipment utilization rate, product maintenance days, asset turnover rate, product compliance rate, product return rate, safety productivity, aftersales service, logistics loss rate, product quality qualified rate of upstream enterprises

Natural risk rate (such as fire, earthquake, and flood), economic risk rate (such as market supply and

Risk level indicator demand, finance, and financing), political risk probability (such as policy and reform risk), technical risk probability (such as technology and process improvement risk and calculation distortion risk), equipment failure risk probability (such as accident risk and downtime risk)

design of indicators to fully reflect the performance of all aspects of the enterprise, factors that influence enterprise intelligent financial management decision are divided into economic (financial) effect, environmental effect, and social effect. Therefore, before comprehensive evaluation of each scheme of the intelligent financial management project, the index system that can fully reflect the merits and demesits of the intelligent financial management scheme should be established first, as shown in Table 1.
With a clear hierarchy, clear thinking, simple calculation, can reflect both objective factors and subjective factors, the first subjective determination of the relative weight between the indicators, on this basis and then through mathematical derivation and quantification, so as to calculate more scientific and accurate than only based on subjectivity. Therefore, analytic hierarchy process is widely used in many subjects.

Step one: decompose into a hierarchy. 


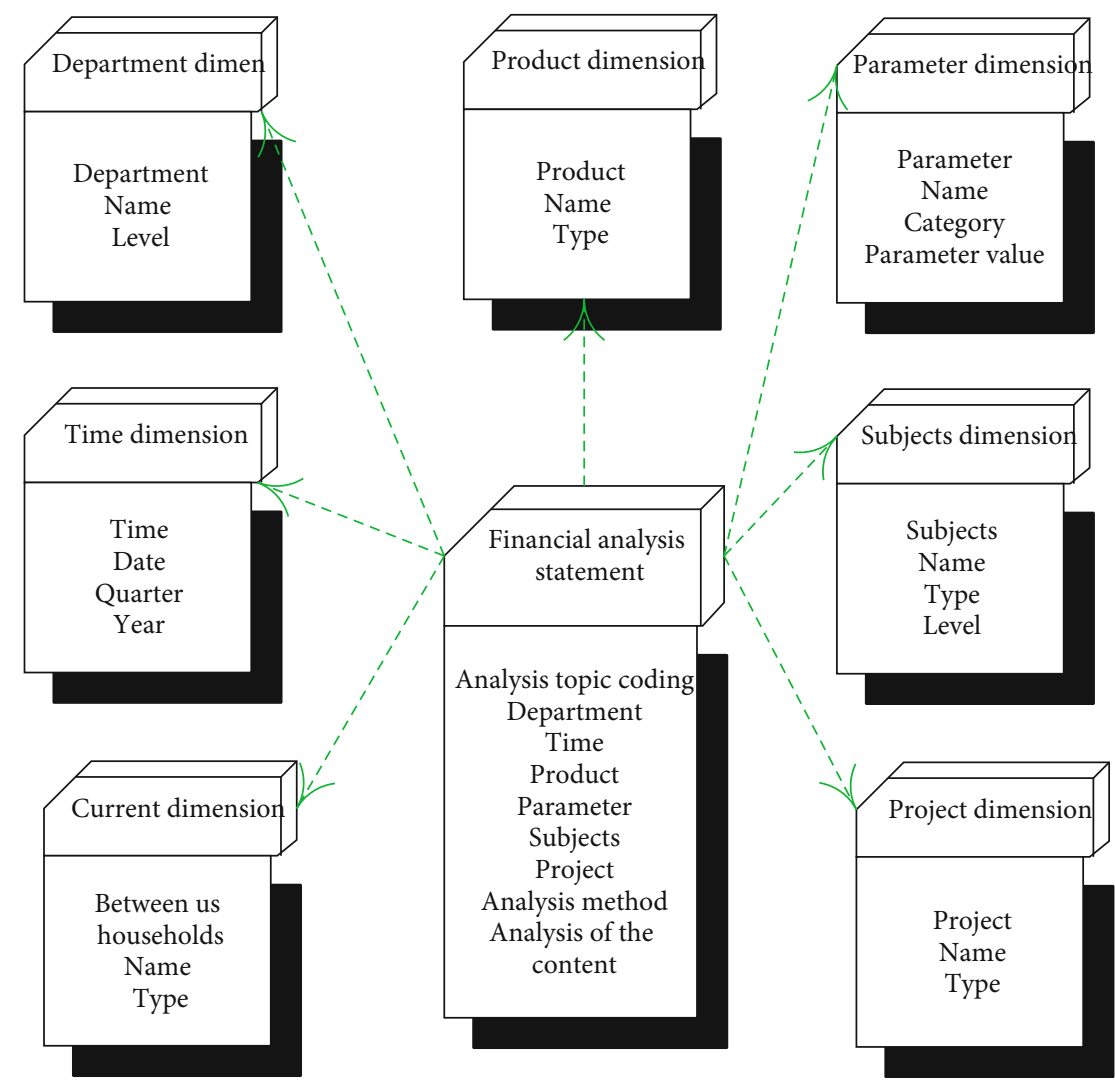

FIgURE 3: Data mining entity attributes of intelligent financial system.

Firstly, the elements are divided into several groups according to their attributes and then decomposed into different levels by the dominant relationship to form a hierarchy structure. There are basically three levels. The highest level, or goal level, is the result or ideal goal to be achieved in order to solve the actual problem. In the middle layer or criterion layer, its elements for the realization of the target layer involved in the intermediate links, the layer is allowed to have multiple layers, including rules to consider and criteria. The lowest layer, or measure layer, is also called scheme layer, which provides various optional measures and schemes to achieve goals.

The second step is to construct the contrast matrix.

The hierarchy can clearly reflect the relationship between the elements in the upper and lower layers. But the complex problem cannot find the ideal result from the hierarchy structure and must use the method of comparison to get the weight. The method of comparison is to compare the two elements, $u$, with UI $(I, j=1,2, \cdots . . N)$. Find out what is important and to what extent and quantify it.

Step 3: predict.

There are many similarities between classification, estimation, and prediction. These are three confusing concepts, and it is not easy to distinguish them. Classification and estimation are for current conditions, while the purpose of prediction is to determine the future output value of an attribute or some attribute. In order to achieve this goal, it is necessary to build a trend model in the current data and use the model to predict the future. The development process of some data

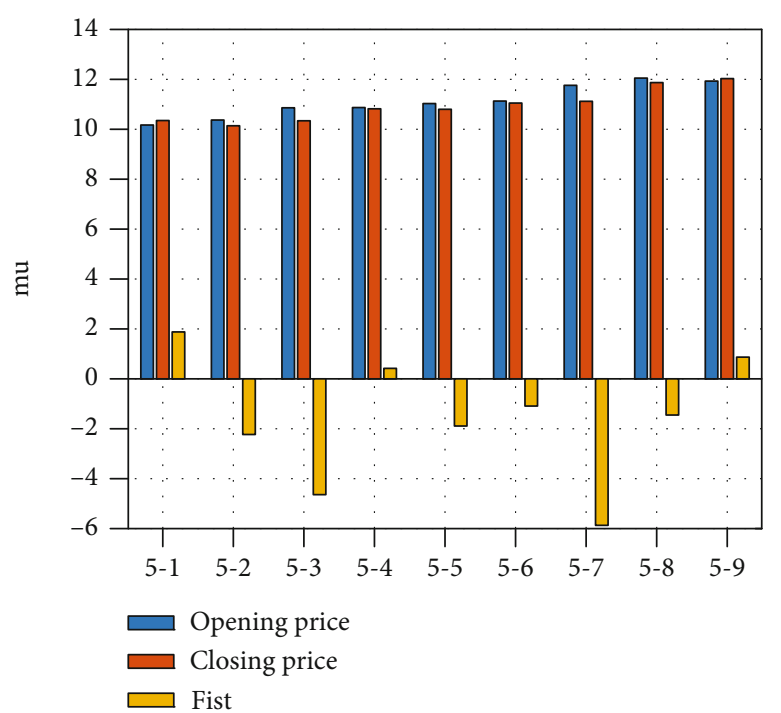

FIGURE 4: Data format of intelligent financial management system.

is a cyclic process or a traceable process, so as long as the development model of this kind of data is established, and its future trend can be predicted. After determining the attributes that need to be predicted, the statistical analysis method is used to analyze the data centrally and judge the trend of attribute values according to similar data. Common prediction models include predicting the rate of return of a 


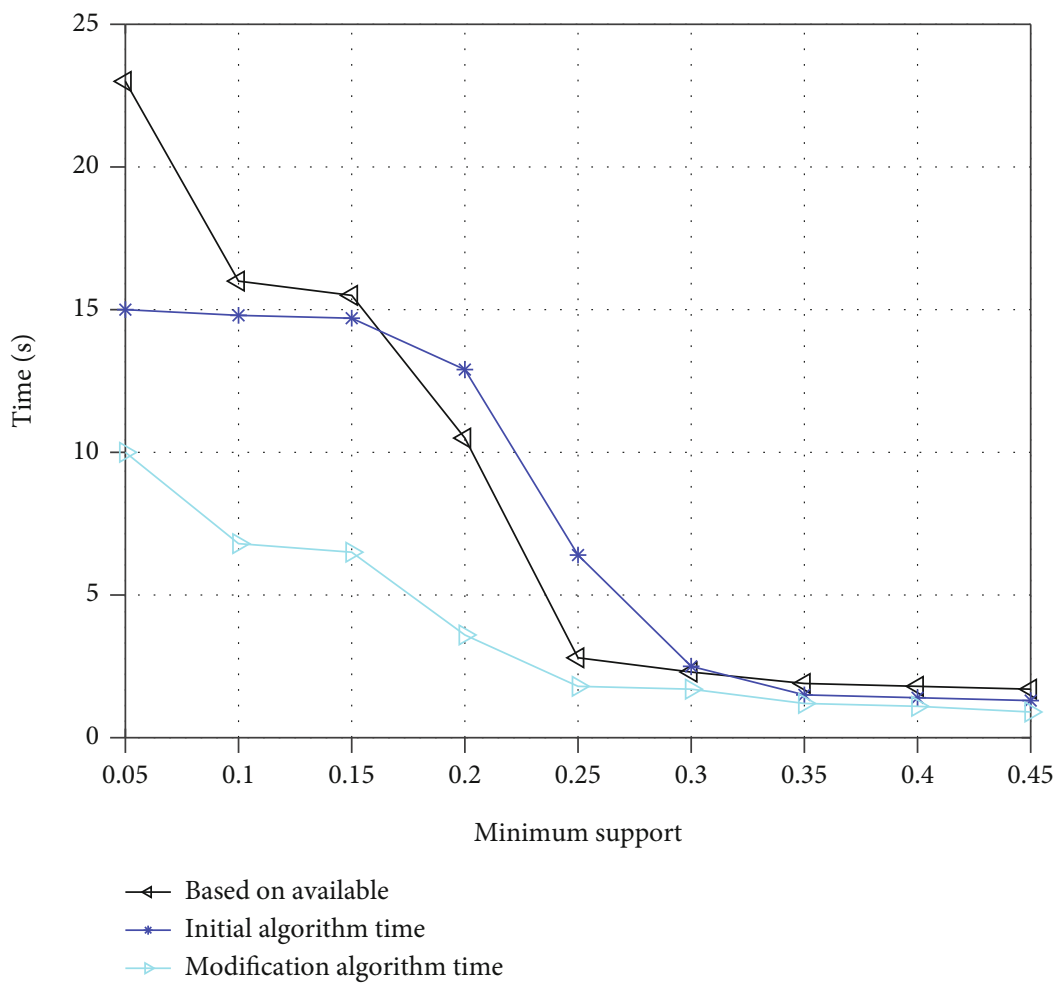

Figure 5: Time comparison diagram.

certain intelligent financial management, predicting the demand for products in the next quarter, and predicting the price trend of production materials.

The fourth step is association analysis.

Correlation refers to the relationships between objects or the rules that exist between objects. Association analysis is widely used in the analysis of commodity transaction data; so, it is also called shopping basket analysis, which refers to the mining of commodity information with correlation from the sales data of products. These analysis results are used to assist product distribution, display design, promotion methods, and other decisions. Of course, association analysis is not limited to the analysis of product sales data, and it can also be used to analyze the causes of certain results.

Enterprise financial analysis system based on data mining is mainly for enterprise leaders, financial department managers, financial analysts, and engaged in intelligent analysis of enterprise financial management and other fields of financial management. Through the investigation and analysis of the needs of the enterprise management, intelligent financial managers, and financial analysts, the conceptual structure relationship between the potential entities in each functional module of the enterprise financial analysis system is further clarified, which lays a good foundation for the logical structure and physical structure design of the database. Financial analysis system based on data mining mainly includes enterprise financial management, asset inventory management, asset allocation management, asset depreciation and verification, asset maintenance management, and other functions. E-rc entity relation can best reflect the relationship between entities and attributes in the conceptual structure. Therefore, e-R diagram will be used in this paper to represent the relationship between entity attributes in the conceptual structure, as shown in Figure 3.

\section{Example Verification}

The data of this paper comes from the CSMAR of the Library, which is based on the accurate database of the Company and covers the data of stock market, bond market, fund market, foreign exchange market, and so on. 36 stocks listed companies were randomly selected from the database to extract the data from May 1, 2010 to May 9, 2011. First of all, we preprocessed the data and found that some stocks had incomplete transaction data. For example, some stocks were newly listed in 2011. After eliminating the incomplete stock, the specific field is processed. Since only the daily opening price can be obtained, the data is preprocessed first, and the daily rise degree is calculated according to the formula of today's rise degree $=$ (today's closing price - yesterday's closing price)/yesterday's closing price. In the programming implementation, first judge whether the degree of increase is greater than zero, in order to take out the increase data. Finally, there are 25 stocks left with 6,100 pieces of data. The data format is shown in Figure 4. If its less than 0, it means today's growth is negative.

In order to verify the improved effect of the algorithm, the data is first run by the basic algorithm. The support degree of the first selection is 0.01 . In general, the smaller the support degree is, the more candidate sets are, and the higher the time cost of operation is, which is convenient for better comparison. When the minimum support 


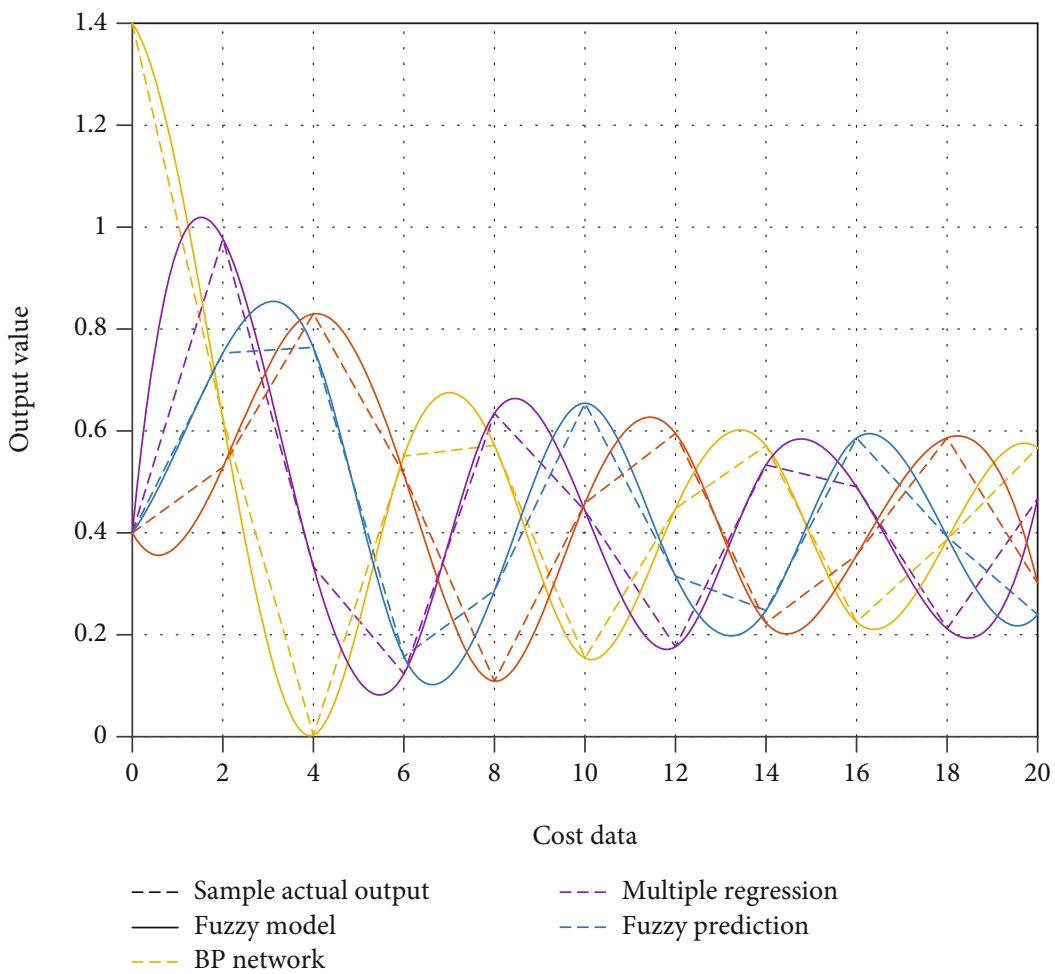

FIGURE 6: Fitting analysis of intelligent financial sample cost data and prediction value of optimized fuzzy model.

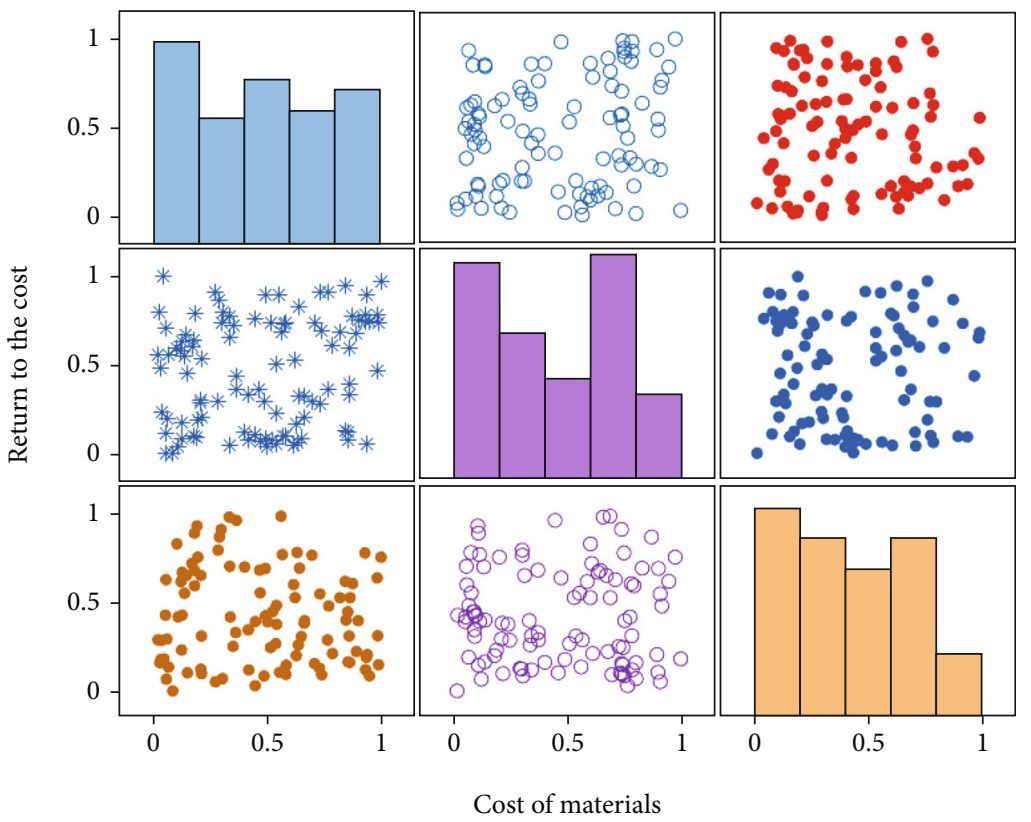

FIgURE 7: Data mining process and results of intelligent financial management system.

continues to increase, the running time of the basic algorithm, the algorithm after preliminary improvement, or the algorithm after further improvement has a nonincreasing trend. The smaller the minimum support is, the number of frequent candidate sets and frequent sets in each stage is larger, and the number of comparisons to be made also increases correspondingly. Therefore, the running time decreases with the increase of the minimum support. In order to have a more intuitive judgment, the effect of the running time of the three algorithms in the same picture is shown in Figure 5.

It can be seen from Figure 5 that the initial improved algorithm takes longer than the basic algorithm under certain minimum support degrees. However, considering 
the average time of the whole series, the initial modified algorithm is still better than all the minimum support degrees of the basic algorithm, and the operation efficiency is higher than the basic algorithm and the initial improved algorithm.

The proposed method was compared with BP neural network and multiple linear regression (MLR) to verify the performance of the optimized fuzzy model. Multiple linear regression method is a kind of multivariate statistical technique, which builds the relationship between independent variables and dependent variables by fitting observation data with linear formula. Multiple linear regression can be considered as an extended simple linear regression method. BP network is an important method in neural network, which is widely used in various fields. A typical BP network usually has three layers. The input layer receives input data from the outside and performs calculation at the neuron nodes of the hidden layer and output layer until the output value satisfying the constraints is obtained and terminated.

During the training of sample cost data, test analysis is also carried out, as shown in Figure 6. In other words, leave-one-out crossvalidation (LOOCV) is firstly adopted, in which each sample cost data is individually taken as the validation set, and the remaining $\mathrm{N}-1$ sample cost data is taken as the training set. The "leave one" crossvalidation method can avoid data overfitting to a large extent; then, in each crossvalidation round, the validation set output of multiple linear regression method, BP neural network, and optimized fuzzy model is applied, respectively, to fit the actual output of the sample.

After combining the outlier detection algorithm based on weighted Euclidean distance sum, the final data mining process and results of the intelligent financial product are shown in Figure 7. The outlier rate is set to 5\% in the example.

\section{Conclusion}

Puts forward the solution, a new type of decision support system of object-oriented software development technology has made great progress, there are many ideas such as software system structure, design patterns, and to form a new train of thought and technology method, and the emergence of these technologies for the development of intelligent decision support system (IDSS) provides a more convenient tool, further shortens the software development cycle and enhances the software scalability. First, discuss decision support system and intelligent decision support system research status and some related problems and then further study the decision support system and intelligent decision support system of system structure and system characteristics and combining the relevant theory research identified in the study of the intelligent smart financial management support system of the basic concepts and characteristics, as well as the deficiencies existing in the design of the system, and the system architecture of enterprise intelligent financial management support system based on data mining technology is established. First to introduce the relevant basic knowledge of data mining, such as the definition, the process of data mining, and data mining algorithms for mining, through the analysis and summary of intelligent data mining algorithm, is found that its deficiencies and put forward the improved algorithm, aiming at the shortcomings of the algorithm and the improved algorithm on the mining experiment, and experiment results show that has certain advantages.

\section{Data Availability}

The data used to support the findings of this study are included within the article.

\section{Conflicts of Interest}

All the authors do not have any possible conflicts of interest.

\section{References}

[1] Y. Chen, S. Zhao, and Y. Zhou, "Research on intelligent agricultural planting system based on internet of things technology," Journal of Computer and Communications, vol. 6, no. 6, pp. 54-60, 2018.

[2] C. Xiang and B. Li, "Research on ship intelligent manufacturing data monitoring and quality control system based on industrial internet of things," The International Journal of Advanced Manufacturing Technology, vol. 107, no. 3-4, pp. 983-992, 2020.

[3] Z. M. Zhu, F. Q. Xu, and X. Gao, "Research on school intelligent classroom management system based on Internet of Things," Procedia Computer Science, vol. 166, pp. 144-149, 2020.

[4] X. Lv and M. Li, "Application and research of the intelligent management system based on Internet of Things Technology in the era of big data," Mobile Information Systems, vol. 2021, no. 16, Article ID 6515792, 36 pages, 2021.

[5] Y. Cui, "Intelligent recommendation system based on mathematical modeling in personalized data mining," Mathematical Problems in Engineering, vol. 2021, no. 3, Article ID 6672036, 11 pages, 2021.

[6] C. Geng, Y. Xu, and N. Metawa, "Intelligent financial decision support system based on data mining," Journal of Intelligent and Fuzzy Systems, vol. 2, pp. 1-10, 2021.

[7] I. Abunadi, "Characteristics of electronic integrated system and trust in the provider of service," International Journal of Computer Applications, vol. 132, no. 4, pp. 23-31, 2015.

[8] Y. Deng, W. Sun, M. Chen, and Y. Yang, "Knowledge management and e-learning in virtual learning community based on social network analysis," Library Hi Tech, vol. 37, no. 4, pp. 906-917, 2019.

[9] L. Lu and J. Zhou, "Research on mining of applied mathematics eucational resources based on edge computing and data stream classification," Mobile Information Systems, vol. 2021, no. 7, Article ID 5542718, 1238 pages, 2021.

[10] L. Jia, "Intelligent data mining algorithm application in enterprise financial management strategy optimization: a Ecommerce perspective," International Journal of Security and its Applications, vol. 10, no. 7, pp. 157-168, 2016.

[11] Q. Qin, S. Cheng, Q. Zhang, Y. Wei, and Y. Shi, "Multiple strategies based orthogonal design particle swarm optimizer for numerical optimization," Computers \& Operations Research, vol. 60, pp. 91-110, 2015.

[12] Z. Li and J. Wang, "An optimization application of artificial intelligence technology in enterprise financial management," Boletin Tecnico/technical Bulletin, vol. 55, no. 11, pp. 83-89, 2017. 
[13] Q. Wang and B. Zhang, "Research and implementation of the customer-oriented modern hotel management system using fuzzy analytic hiererchical process (FAHP)," Journal of Intelligent and Fuzzy Systems, vol. 40, no. 4, pp. 8277-8285, 2021.

[14] D. Jahed Armaghani, E. Tonnizam Mohamad, M. Hajihassani, S. V. Alavi Nezhad Khalil Abad, A. Marto, and M. R. Moghaddam, "Evaluation and prediction of flyrock resulting from blasting operations using empirical and computational methods," Engineering with Computers, vol. 32, no. 1, pp. 109-121, 2016.

[15] X. Gong, Z. Wang, and L. Wang, "Research on financial early warning model for papermaking Enterprise based on particle swarm K-means algorithm," Paper Asia, vol. 34, no. 6, pp. 41-45, 2018.

[16] S. Zhai, "Research on enterprise financial management and decision making based on decision tree algorithm," Boletin Tecnico/Technical Bulletin, vol. 55, no. 15, pp. 166-173, 2017.

[17] Y. Wei, "Research on design and realization of enterprise accounting management platform based on data mining," International Journal for Engineering Modelling, vol. 31, no. 1, pp. 265-272, 2018.

[18] L. Wang, Y. Liu, and J. Wu, "Research on financial advertisement personalised recommendation method based on customer segmentation," International Journal of Wireless and Mobile Computing, vol. 14, no. 1, pp. 97-121, 2018.

[19] C. Jun and P. Jin, "Financial management and decision of decision tree algorithm based on data mining," IPPTA: Quarterly Journal of Indian Pulp and Paper Technical Association, vol. 30, no. 8, pp. 70-74, 2018.

[20] R. Geng, I. Bose, and X. Chen, "Prediction of financial distress: an empirical study of listed Chinese companies using data mining," European Journal of Operational Research, vol. 241, no. 1, pp. 236-247, 2015.

[21] K. Lv, "Study on pharmaceutical database management based on data mining technology," Journal of Information and Computational Science, vol. 12, no. 8, pp. 2979-2986, 2015.

[22] H. He, Y. Hong, W. Liu, and S. A. Kim, "Data mining model for multimedia financial time series using information entropy," Journal of Intelligent and Fuzzy Systems, vol. 39, no. 4, pp. 5339-5345, 2020.

[23] H. Sun, Z. Yao, and Q. Miao, "Design of macroeconomic growth prediction algorithm based on data mining," Mobile Information Systems, vol. 2021, no. 7, Article ID 2472373, 138 pages, 2021.

[24] Y. Lu, "Financial accounting intelligence management of internet of things enterprises based on data mining algorithm," Journal of Intelligent and Fuzzy Systems, vol. 37, no. 5, pp. 5915-5923, 2019.

[25] S. Lessmann, B. Baesens, H. V. Seow, and L. C. Thomas, "Benchmarking state-of-the-art classification algorithms for credit scoring: an update of research," European Journal of Operational Research, vol. 247, no. 1, pp. 124-136, 2015.

[26] K. P. Nguyen and V. Nguyen, "Analysis of weather information system in statistical and rough set point of view," Studies in Computational Intelligence, vol. 572, pp. 97-106, 2015. 